\title{
Satisfaccion del control \\ y alivio del dolor en usuarios \\ postoperados hospitalizados
}

\author{
Control and pain relief satisfaction \\ in postoperative hospital users
}

\begin{abstract}
Enf. Ma.Esmeralda Leal Cavazos* Enf. Mirna Daleth Mendoza Nájera* Enf. Mirna Daleth Mendoza Nájera* Lic.Norma Esther Hernández Vázquez*, M. C. E. Hermelinda Ávila Alpirez** M. C.E. Barbarita Frías Reyna* M. C. A. Gloria Nelly Gracia Castillo*

*Estudiante del Curso Pos-Básico de Enfermería Quirúrgica de la Unidad Académica Multidisciplinaria Matamoros- UAT Enfermera del Hospital General " DR. Alfredo Pumarejo de H. Matamoros, Tamaulipas

**Profesora de Carrera "G" de la Unidad Académica Multidisciplinaria Matamoros-UAT ( UAMM_UAT)
\end{abstract}

\section{Resumen}

Introducción: Es una evidencia innegable que el dolor es uno de los principales problemas que enfrenta el usuario post quirúrgico en el ámbito hospitalario, sin embargo, es poco lo que sabemos sobre la satisfacción del usuario con la atención recibida que es clave en la mejora de la calidad asistencial.

El propósito de la investigación fue conocer la Satisfacción del control y Alivio del Dolor en Usuarios Postoperados hospitalizado en las instituciones de salud de $\mathrm{H}$. Matamoros, Tamaulipas.

Metodología. El diseño fue de tipo descriptivo, trasversal. El muestreo fue no probabilístico por conveniencia la muestra estuvo conformada por de 30 usuarios hospitalizados Postoperados. Se utilizó el instrumento.de la Asociación

Introduction: The purpose of the investigation was to know the Satisfaction and the experience of the control and Relief of the Pain in post operated users and hospitalized in the institutions of health of $\mathrm{H}$. Matamoros, Tamaulipas.

Methodology. The design was of descriptive, traverse type. The sampling was probability for convenience; the sample was conformed out of 30 hospitalized post operated users. The instrument of the American Association of the Pain was used, being this a validated and reliable instrument.

Results. Descriptive statistics were used, 73.3\% corresponds to female, and the age of those interviewed was of 31-35 in
Americana del Dolor, siendo este un instrumento validado y confiable.

. Se utilizó estadísticas descriptivas, Resultados El 96.7\% del los usuario mostraron estar totalmente satisfecho en el control y alivio del dolor.

Discusión y Conclusiones Los resultados obtenidos mostraron algo de dolor de acuerdo a su experiencia en el posoperatorio en los sujetos de estudio. Respecto a la satisfacción del paciente al control y alivio del dolor se encontró que fue totalmente satisfecho, así como también su nivel de satisfacción con respecto al cuidado de enfermería considerando al paciente como una persona que experimenta el dolor.

Palabras claves: Satisfacción, control y alivio del dolor.

\section{Abstract}

$23.3 \%$, in regards to the marital status most pointed out to be married or have moved in. $96.7 \%$ showed to be completely satisfied with the control and relief of the pain. The experience of the pain of those subjects of study was of $63.3 \%$ referring some pain.

Discussion and Conclusions. Galician et al agree with the data obtained regarding the users satisfaction of the control and relief of the pain.Contrary to Peñarrieta's report et al, when having experienced the worst pain.

Key words: Satisfaction, control and pain relief 


\section{INTRODUCCIÓN}

El sistema mexicano de salud enfrenta problemas tanto en el componente técnico como en la calidez de los servicios, estos problemas de calidad además, son claramente percibidos como prioritarios por la población mexicana, los problemas de calidad no son privativos del sector público

En conformidad el programa nacional de salud 20012006, menciona que los servicios públicos y privados que se ofrecerán serán de alta calidad técnica y deberán satisfacer las expectativas de los usuarios, los habrán de proporcionar prestadores mas capacitados que harán uso de intervenciones basados en evidencia científica, estos servicios promoverán el desarrollo profesional, la satisfacción de los usuarios y el respeto a los derechos humanos. $^{1}$

${ }^{2} \mathrm{El}$ dolor es una experiencia humana compleja y frecuentemente infravalorada, es el síntoma mas frecuente en el manejo clínico o quirúrgico de las enfermedades que afectan al ser humano, pese a los adelantos científicos médicos y tecnológicos, el paciente sufre innecesariamente por un dolor que debiera estar mas controlado en el medio hospitalario.

El dolor postoperatorio es aquel que aparece como consecuencia del acto quirúrgico constituye aún un problema no resuelto en los pacientes quirúrgicos ${ }^{3}$ aunque durante las últimas dos décadas se han producido avances considerables referentes al conocimiento de la fisiopatología del dolor agudo, la introducción de nuevos fármacos y el desarrollo de nuevas técnicas y modos de administración. Sin embargo, estos hallazgos no han producido una mejora objetivable en el tratamiento del dolor postoperatorio, siendo su incidencia similar a lo largo de estudios realizados en diferentes países durante los últimos años. ${ }^{4}$

Zaragoza cita a la (Sociedad Española del Dolor [S.E.D] ) Donde reporta la incidencia de dolor postoperatorio en hospitales españoles y los resultados mencionan que los pacientes presentaban dolor en las primeras horas del postoperatorio. ${ }^{5}$

El control satisfactorio del dolor post operatorio es uno de los retos más importantes que permanecen sin resolver en el ámbito quirúrgico, lo que motiva un fuerte impacto en los pacientes y en el sistema sanitario en su conjunto.

Es una evidencia innegable que la el dolor es uno de los principales problemas que enfrenta el usuario post quirúrgico en el ámbito hospitalario, sin embargo, es poco lo que sabemos sobre la satisfacción del usuario con la atención recibida que es clave en la mejora de la calidad asistencial. $^{6}$

El profesional de enfermería como ente proveedor de servicios de salud, realiza un acto personal ético, creativo y solidario a las personas en el proceso salud-enfermedad en el que se precisa una atención adecuada con la finalidad de fortalecer emocionalmente al paciente proporcionando así cuidados de enfermería de calidad. El dolor postoperatorio es necesidad de tratamiento rápido y eliminar la causa que lo provoca, la satisfacción de los pacientes con el personal medico y de enfermería, cuantificar el tiempo y efectividad de los analgésicos. ${ }^{7}$

\section{Objetivo General}

Describir la satisfacción del control y alivio en la experiencia del dolor en usuarios postoperados hospitalizados

\section{METODOLOGÍA \\ Diseño del Estudio}

El diseño fue de tipo descriptivo trasversal ${ }^{8}$ El muestreo fue probabilístico por conveniencia Criterios de inclusión: usuarios postoperados, hospitalizados de 24 horas, sin complicaciones, bien orientado, ambos sexos, edad entre 20 y 66 años. El tamaño de la muestra fue 30 usuarios hospitalizados.

\section{Procedimiento de Recolección de Datos}

Una vez obtenida la autorización de las instituciones de salud Se procedió a pasar a sus cubículos para solicitarle su participación y explicándole el objetivo del estudio, se le dio a conocer el consentimiento informado una vez firmado se procedió a aplicar el instrumento. Previamente a lo anterior se corroboraron los criterios de inclusión a través de preguntas sencillas.

Se estuvo atento a cualquier signo de alarma dolor o negación para suspender la aplicación, en este caso fue necesario en un solo paciente hacer una pausa y posteriormente se continuo con el cuestionario.

\section{Instrumento}

Para medir la satisfacción del control y alivio del dolor en usuarios post operados hospitalizados se utilizó un cuestionario adaptado de la Asociación Americana del Dolor elaborado por Janeth McNaill. 


\section{Consideraciones Éticas}

La presente investigación se apego a lo dispuesto en Reglamento de la Ley General de Salud en materia de Investigación para la salud. ${ }^{9}$

\section{Análisis de Resultados}

Los resultados obtenidos se procesaron por el paquete estadístico Statistical Package for the Social Sciences (SPSS), versión 17 para Windows, Se utilizó estadísticas descriptivas para describir las características de la muestra y variables de estudio; también el coeficiente de confiabilidad Alpha de Cronbach permitió determinar la consistencia interna del instrumento.

\section{RESULTADOS}

El instrumento de la Asociación Americana del Dolor , es valido y confiable. ya que su consistencia interna fue de .84

Los datos Sociodemográficos del estudio reportaron que el $73.3 \%$ de la muestra corresponde al sexo femenino, en cuanto a la edad el 23.3\% fluctúan entre 31 y 35 años y estado marital de los encuestados fue en un $90 \%$ casados o en unión libre. (Tabla 1)

La experiencia del dolor que refirieron los sujetos de estudio de acuerdo a la escala visual analógica fue de algo de dolor en las variables de dolor presente en un 63.3\% así como también el peor dolor que a tenido en 24 horas el $70 \%$ y en la intensidad usual en las ultimas 24 horas fue $86 \%$.(Tabla 2 ).

\section{Tabla 1}

\section{Datos Sociodemográficos}

\begin{tabular}{lcc}
\multicolumn{1}{c}{ Características } & $\mathbf{f}$ & \% \\
\hline Sexo & 22 & 73.3 \\
Femenino & 8 & 26.7 \\
Masculino & & \\
Edad & 7 & 23.3 \\
$31-35$ & 5 & 16.7 \\
$36-40$ & 4 & 13.3 \\
$46-50$ & & \\
Estados Civil & 27 & 90.0 \\
Casado o unión libre & 3 & 10.0 \\
Soltero, viudo o divorciado & \\
Fuente: Cedula de datos Sociodemográficos $\mathrm{n}=30$ & \\
\end{tabular}

(Tabla 3) La satisfacción personal en cuanto al control y alivio del dolor respecto al cuidado que las enfermeras/ os le proporcionaron el $96.7 \%$ mostró estar totalmente satisfecho, el 93.3\% reportaron el cuidado general que recibieron por su dolor fue totalmente satisfecho

\section{DISCUSIÓN}

De acuerdo a los resultados obtenidos en este estudio los sujetos reportaron algo de dolor de acuerdo a su experiencia en el postopertorio Estos resultados concuerdan por lo reportado por Gallego en donde menciona que sus pacientes refirieron dolor moderado, contrariamente a lo reportado por Peñarrieta donde señala que los pacientes experimentaron el peor dolor.

Respecto a la satisfacción del paciente en cuanto al control y alivio del dolor se encontró que fue totalmente satisfecho, así como también su nivel de satisfacción con respecto al cuidado de enfermería considerando al paciente como una persona que experimenta el dolor. Opuesto a lo que menciona Peñarrieta en donde refirieron insatisfacción en cuanto a los cuidados de enfermería así como al control y alivio del dolor

\section{CONCLUSIONES}

Se pudo observar que los usuarios manifestaron la experiencia del dolor en cuanto de intensidad fue en un mayor porcentaje seguido de el peor dolor usual en las últimas 24 horas

La satisfacción del usuario en cuanto cuidado que las enfermeras/os les proporcionaron fue totalmente satisfecho.

\section{RECOMENDACIONES}

Mejorar la comunicación entre la relación enfermera/ paciente respecto a la información del dolor posquirúrgico.

Gestionar a las instancias correspondientes para la existencia de la diversidad de fármacos que permitan el control y alivio del dolor.

El personal de Enfermería puede utilizar otros métodos diferentes para el control y alivio del dolor, tales, como, compresas, masaje, relajación otras alterativas de contribuir a satisfacción con la educación que reciba el usuario.

Seguir explorando el fenómeno de estudio ampliando más la muestra para lograr comparaciones entre la

satisfacción del control y alivio en la experiencia del dolor en usuarios postoperados hospitalizados 


\section{Tabla 2}

Experiencia con el dolor

\begin{tabular}{|c|c|c|c|c|c|c|}
\hline \multirow[t]{2}{*}{ Experiencia } & \multicolumn{2}{|c|}{$\begin{array}{c}\text { 0-3 } \\
\text { Ningún dolor }\end{array}$} & \multicolumn{2}{|c|}{$\begin{array}{c}\text { 4-7 } \\
\text { Algo de dolor }\end{array}$} & \multicolumn{2}{|c|}{$\begin{array}{c}8-10 \\
\text { El peor dolor } \\
\text { imaginable }\end{array}$} \\
\hline & $f$ & $\%$ & $\mathbf{f}$ & $\%$ & $f$ & $\%$ \\
\hline Dolor presente & 9 & 30 & 19 & 63.3 & 2 & 6.7 \\
\hline $\begin{array}{l}\text { Peor dolor que ha tenido en las últimas } \\
24 \text { horas }\end{array}$ & 1 & 3.3 & 21 & 70 & 8 & 26. \\
\hline $\begin{array}{l}\text { Intensidad usual de dolor en las últimas } \\
24 \text { horas }\end{array}$ & 1 & 3.3 & 26 & 86. & 3 & 10.0 \\
\hline
\end{tabular}

\section{Tabla 3}

Satisfacción personal en relación a control y alivio del dolor

\begin{tabular}{lcc}
\multicolumn{1}{c}{ Nivel de satisfacción con respecto a: } & \multicolumn{1}{c}{\begin{tabular}{c}
$\mathbf{8 - 1 0}$ \\
\multicolumn{1}{c}{ El alivio que sintió del dolor }
\end{tabular}} & \multicolumn{1}{c}{ Totalmente satisfecho } \\
\cline { 2 - 3 } & $\mathbf{2 0}$ & $\mathbf{6 6 . 7}$ \\
\hline El cuidado que las enfermeras /os le dieron por su dolor. & 29 & 96.7 \\
La atención que le dieron como persona con dolor. & 27 & 90 \\
El cuidado que el doctor le dio por su dolor & 27 & 90 \\
$\begin{array}{l}\text { Que tan rápido las enfermeras/os actuaron cuando pidió medicinas o } \\
\text { ayuda por su dolor } \\
\text { La manera de cómo sus familias/amigos fueron incluidos en el control de } \\
\text { su dolor } \\
\text { El cuidado general que recibió por su dolor }\end{array}$ & 25 & 83.3 \\
\hline
\end{tabular}

\section{REFERENCIAS BIBLIOGRÁFICAS}

1 Encuesta Nacional de Salud, Programa Nacional de Salud 2001-2006 p 2

2 Martin A, De la Riva D, Sanz T M; Urraca V, García V, Azpiazu M, Visión del dolor posoperatorio no controlado. Revista Rol de Enfermería 2002 Enero 18; (consultados 07/04/2006on line) Disponible en http:www.ocenf.org/ Cantabria/inves.htm.

3 Carr DB, Goudas LC. Acute pain. The Lancet 1999; (353), 2051-2058.

4 Allvin R, Brasseur L, Crul B, Dhal J, Lehmann K, Nicosia F, Puig MM, Rawal N, Richards S, Wheatley R. European Minimum standards for the management of postoperative pain. EuroPain Task Force. Pegasus Helthcare Intl, UK, 1998;(45) 465-470.

5 Zaragoza G. F, Landa G.I, Larrainzar G.R, Monino R.P, De la Torre L.R, Dolor posoperatorio en España, Julio 2005.

6 Peñarrieta DC,I., Del Ángel RE, García BM, Vergel CS, González SF, Feletti G, Zavala RMR, Rev. Manejo del dolor y la satisfacción del pacientes post operados hospitalizados Desarrollo Científico de Enfermería, 2006 (14), 20-26.

7 GallegoJl, Rodríguez MR., Vázquez JC, Gil M, Estimación de la prevalencia e intensidad del dolor postoperatorio y su relación con la satisfacción de los pacientes. Rev. Soc. Esp. del Dolor, 2004.(11),197-202.

8 Polit D, Hungler B, Investigación científica en ciencias de la salud. 6a Ed. México: Mc Graw Hill Interamericana.; 2000 159-191.

9 Secretaría de Salud. (1987). Reglamento de la ley general de salud en materia de investigación para la salud. Recuperado de www.salud.gob.mx el .

\section{DIRECCIÓN PARA CORRESPONDENCIA}

M.C.A. Gloria N. Gracia C.: ggracia3@uat.edu M.C.E. Hermelinda Ávila A.:

avila_alpirez_hermelinda@hotmail.com 